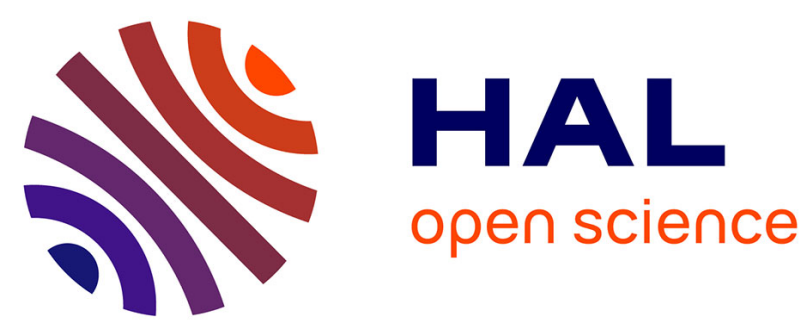

\title{
The practice of recent radiative transfer Monte Carlo advances and its contribution to the field of microorganisms cultivation in photobioreactors
}

\author{
Jeremi Dauchet, Stéphane Blanco, Jean-Francois Cornet, Mouna El-Hafi, \\ Vincent Eymet, Richard A Fournier
}

\section{To cite this version:}

Jeremi Dauchet, Stéphane Blanco, Jean-Francois Cornet, Mouna El-Hafi, Vincent Eymet, et al.. The practice of recent radiative transfer Monte Carlo advances and its contribution to the field of microorganisms cultivation in photobioreactors. Journal of Quantitative Spectroscopy and Radiative Transfer, 2013, 128 (SI), pp.52-59. 10.1016/j.jqsrt.2012.07.004 . hal-01688109

\section{HAL Id: hal-01688109 https://hal.science/hal-01688109}

Submitted on 7 Nov 2018

HAL is a multi-disciplinary open access archive for the deposit and dissemination of scientific research documents, whether they are published or not. The documents may come from teaching and research institutions in France or abroad, or from public or private research centers.
L'archive ouverte pluridisciplinaire HAL, est destinée au dépôt et à la diffusion de documents scientifiques de niveau recherche, publiés ou non, émanant des établissements d'enseignement et de recherche français ou étrangers, des laboratoires publics ou privés. 


\title{
The practice of recent radiative transfer Monte Carlo advances and its contribution to the field of microorganisms cultivation in photobioreactors
}

\author{
Jérémi Dauchet ${ }^{\mathrm{a}, \mathrm{c}, \mathrm{d}, *}$, Stéphane Blanco ${ }^{\mathrm{c}, \mathrm{d}}$, Jean-François Cornet ${ }^{\mathrm{b}}$, \\ Mouna El Hafi ${ }^{\mathrm{e}}$, Vincent Eymet ${ }^{\mathrm{e}}$, Richard Fournier ${ }^{\mathrm{c}, \mathrm{d}}$ \\ ${ }^{a}$ Clermont Université, Université Blaise Pascal, Institut Pascal - UMR 6602, BP 10448, F-63000 Clermont-Ferrand, France \\ ${ }^{\mathrm{b}}$ Clermont Université, ENSCCF, Institut Pascal - UMR 6602, BP 10448, F-63000 Clermont-Ferrand, France \\ ${ }^{\mathrm{c}}$ Université de Toulouse; UPS, INPT; LAPLACE (Laboratoire Plasma et Conversion d'Energie); 118 route de Narbonne, \\ F-31062 Toulouse cedex 9, France \\ ${ }^{\mathrm{d}}$ CNRS; LAPLACE; F-31062 Toulouse, France \\ e Université de Toulouse; Mines Albi; CNRS; Centre RAPSODEE, Campus Jarlard, F-81013 Albi cedex 09, France
}

\begin{abstract}
A B S T R A C T
The present text illustrates the practice of integral formulation, zero-variance approaches and sensitivity evaluations in the field of radiative transfer Monte Carlo simulation, as well as the practical implementation of the corresponding algorithms, for such realistic systems as photobioreactors involving spectral integration, multiple scattering and complex geometries. We try to argue that even in such non-academic contexts, strong benefits can be expected from the effort of translating the considered Monte Carlo algorithm into a rigorously equivalent integral formulation. Modifying the initial algorithm to simultaneously compute sensitivities is then straightforward (except for domain deformation sensitivities) and the question of enhancing convergence is turned into that of modeling a set of well identified physical quantities.
\end{abstract}

Keywords:

Radiative transfer

Monte Carlo

Multiple scattering

Zero-variance

Photobioreactor

Microalgae

\section{Introduction}

During the last 15 years, significant methodological advances have been reported in the field of Monte Carlo simulation of linear transport phenomena, in particular as far as radiative transfer is concerned [1-3]. Among them, attention is here devoted to integral formulation, zerovariance approaches [4,5] and sensitivity evaluations [6], as well as to the question of implementing practically the corresponding algorithms when thinking of realistic systems involving spectral integration, multiple scattering

\footnotetext{
*Corresponding author at: Clermont Université, Université Blaise Pascal, Institut Pascal - UMR 6602, BP 10448, F-63000 Clermont-Ferrand, France. Tel.: + $33(0) 473405317$.

E-mail address: jeremi.dauchet@laplace.univ-tlse.fr (J. Dauchet).
}

and complex geometries. Such techniques all rely on the premise that Monte Carlo algorithms can be designed in more subtle ways than making direct analogies with the statistics of corpuscular transport. But along this line a main advantage of Monte Carlo practice seems to be lost. The fact that standard radiative transfer Monte Carlo codes can be pictured as close translations of well established physical pictures of photon emission, diffusion, scattering, reflection and absorption implies indeed that such codes are easy to design and easy to upgrade toward the representation of additional (or further accurate) physical phenomena. If the use of advanced Monte Carlo techniques implies that these physical pictures are hidden behind complex algorithmic tricks, or that the corresponding codes become so complex that implementation and upgrading are sources of strong practical 
difficulties, then radiative transfer specialists will be reluctant to make an effective use of these techniques, whatever their expected benefits. We try to argue here (and illustrate with a photobioreactor example) that these drawbacks vanish as soon as a strict relationship is made, in an explicit manner, between the considered linear transport Monte Carlo algorithm and the corresponding integral transport formulation. Provided that the relationship is made systematically, whatever the complexity of the problem, the whole reasoning can be made in terms of radiative transfer formulations, keeping all the immediate benefits of available physical pictures. This also implies that the technical aspects that have no direct relation with physical reasoning, in particular the statistical treatments, parallelization, pure geometrical considerations, etc, can all be translated into scientific computation libraries and can be used in quite straightforward manners.

Photobioreactors are processes of biomass production in which photosynthesis is catalyzed by photosynthetic microorganisms in aqueous suspensions constituting non-gray absorbing and anisotropically scattering media. Careful radiation transfer analysis of photobioreactors is identified as the clue for efficient design and efficient operation [7-11]. Monte Carlo methods seem to be appropriate for such an analysis since they allow to tackle the intermediate optical depths and quite complex geometries that are encountered in this context. The present work focuses on a cylindrical reactor prototype (cultivating the micro-algae Chlamydomonas reinhardtii) in which the incident solar light flux density is diluted in the volume of culture thanks to a thousand of light-diffusing optical fibers emitting a quasi homogeneous density flux on the totality of their surface. All details and the corresponding notations are provided in Figs. 1 and 2 (the microorganism density $\eta$ is uniform within the culture volume $\mathcal{V}$, emission is negligible, $k_{a, v}=\eta \sigma_{a, v}$ and $k_{s, v}=\eta \sigma_{s, v}$ are the absorption and scattering coefficients). The following methodological discussions address the estimation of the specific number of photons $A\left(\mathbf{x}_{0}\right)$ absorbed in the photosynthetically active radiation domain $\left[v_{\min }, v_{\max }\right]$, at a location $\mathbf{x}_{0}$ within the microorganism suspension:

$$
\begin{aligned}
A\left(\mathbf{x}_{0}\right) & =\frac{1}{\eta} \int_{v_{\min }}^{v_{\max }} d v \int_{4 \pi} d \omega_{0} k_{a, v} \frac{L_{v}\left(\mathbf{x}_{0},-\omega_{0}\right)}{h v} \\
& =\int_{v_{\min }}^{v_{\max }} d v \int_{4 \pi} d \omega_{0} \sigma_{a, v} \frac{L_{v}\left(\mathbf{x}_{0},-\omega_{0}\right)}{h v}
\end{aligned}
$$

where $L_{v}\left(\mathbf{x}_{0},-\omega_{0}\right)$ is the intensity at $\mathbf{x}_{0}$ in the direction $-\omega_{0}$ at frequency $v$, and $h$ is Planck's constant. $A\left(\mathbf{x}_{0}\right)$ locally determines the kinetics of production within the culture volume since it represents the energy that enters the photosynthesis [7]. The surface productivity of photobioreactors is directly linked to their energetic efficiency which estimation implies to calculate $A\left(\mathbf{x}_{0}\right)$ for more than $10^{6}$ different locations in order to evaluate the production rates integrated over the whole reactor volume (which cannot be done inside the presented Monte Carlo algorithm since the local production rates are non-linear functions of $A\left(\mathbf{x}_{0}\right)$ ). This calculation is then repeated for several sets of operational and design parameters, as a part of design/operation optimization procedures. Therefore, the reduction of the calculation times and the opportunity to estimate sensitivities of $A\left(\mathbf{x}_{0}\right)$ at low calculation costs (in order to accelerate the optimization procedures, thinking for example of a method of steepest descent) both constitute important practical concerns. For these reasons, the simulations presented hereafter are parallelized and make use of computation tools developed by the computer graphics research community for the acceleration of photon tracking in complex

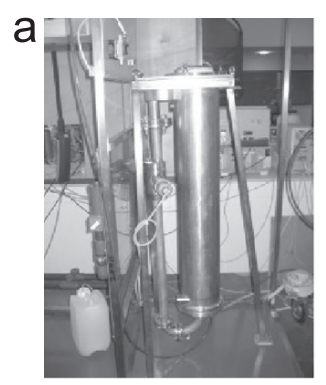

b

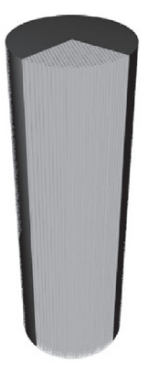

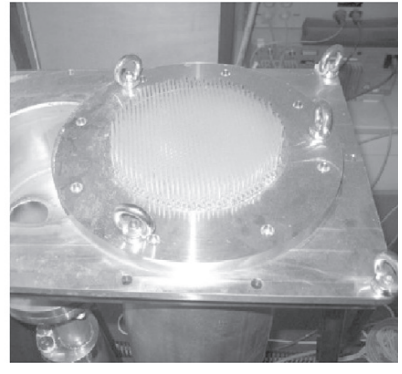

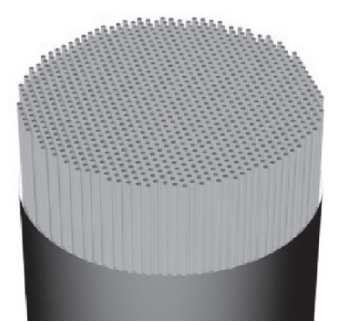

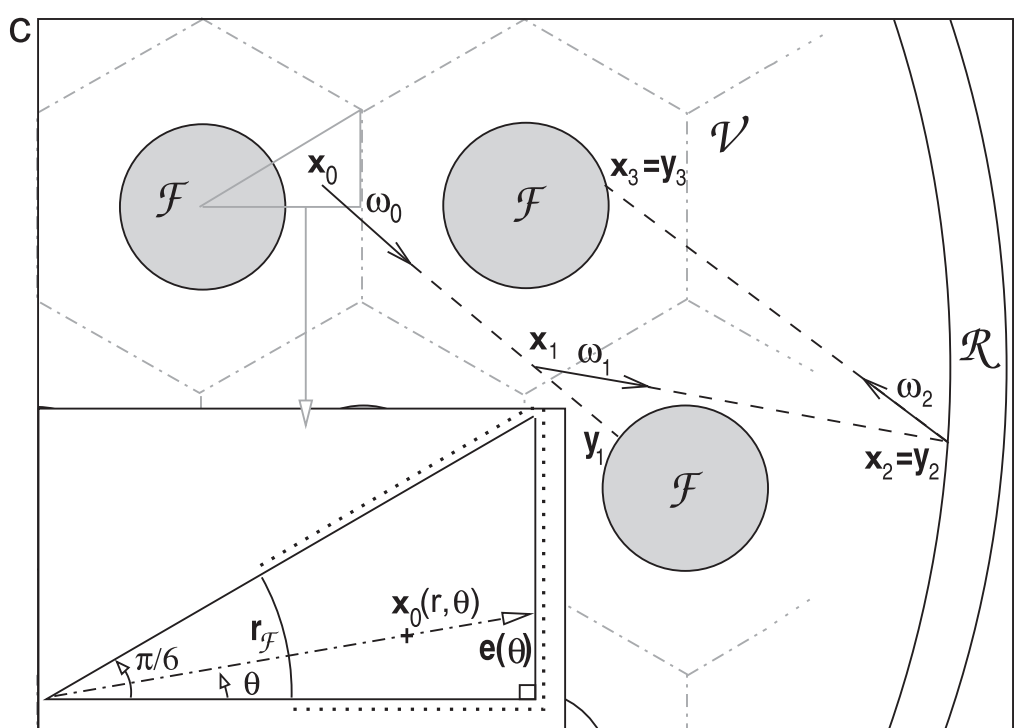

Fig. 1. (a) Prototype of solar volumetrically lightened photobioreactor [8]. (b) EDStar geometry: both the reactor ( $\mathcal{R}$ ) and the 979 light-diffusing optical fibers $(\mathcal{F})$ are cylinders of height $1 \mathrm{~m}$; reactor diameter is $16.5 \mathrm{~cm}$; distance between two fiber axis is $d_{\mathcal{F}}=4.8 \mathrm{~mm}$; fiber radius is $r_{\mathcal{F}}=1.2 \mathrm{~mm}$. $\mathcal{R}$ and $\mathcal{F}$ are diffuse-reflective with uniform reflectivities $\rho^{\mathcal{R}}$ and $\rho^{\mathcal{F}}$ respectively. $\mathcal{F}$ is Lambertian emitting with a uniform surface flux density $\varphi_{v}$. (c) $2 \mathrm{~d}$ hexagonal lattice fiber arrangement; an optical path example within the culture medium $\mathcal{V}$; at the bottom left, description of how the location of $\mathbf{x}_{0}$ is defined around a given fiber $\left(e(\theta)=\left(d_{\mathcal{F}} / 2\right) 1 / \cos (\theta)\right)$. 
a

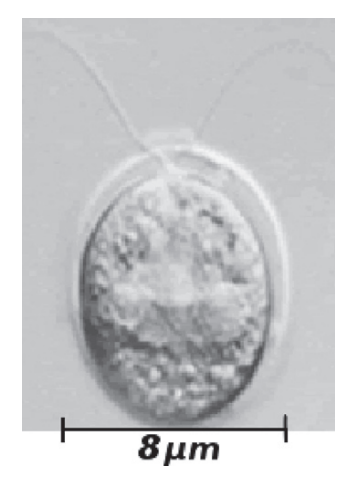

b

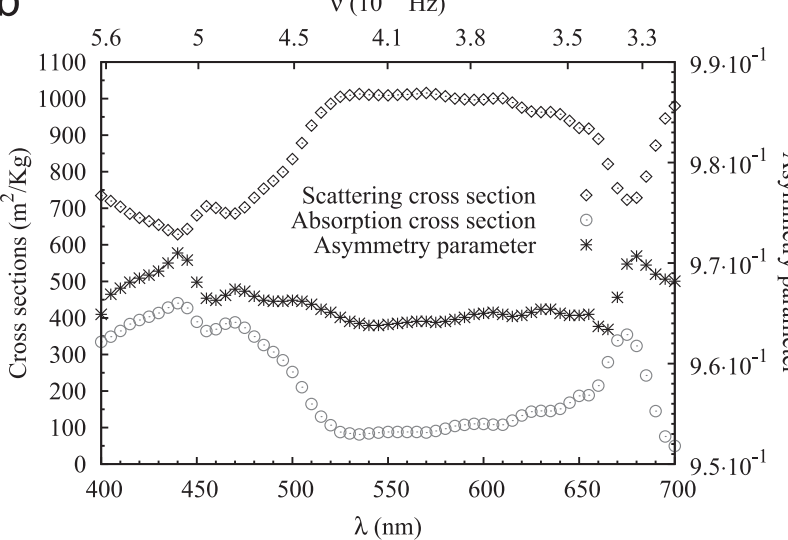

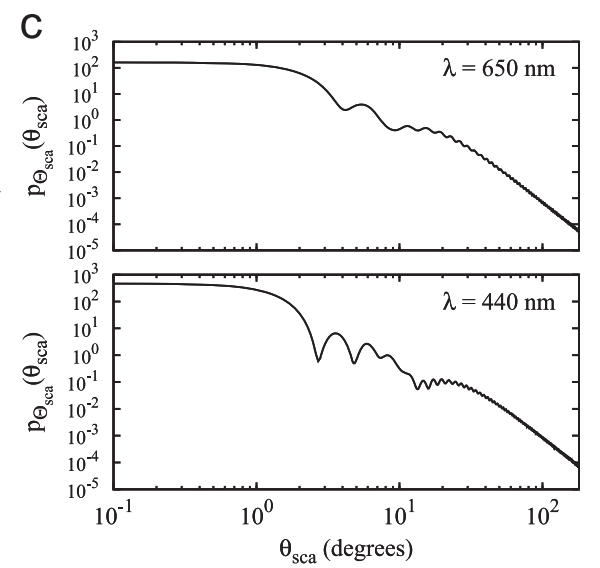

Fig. 2. (a) Chlamydomonas reinhardtii. (b) Radiative properties; the absorption cross-section $\sigma_{a, v}$, the scattering cross-section $\sigma_{s, v}$ and the asymmetry parameter of the single scattering phase function $p_{\boldsymbol{\Omega}, v}^{\mathcal{V}}\left(\boldsymbol{\omega} \mid \boldsymbol{\omega}^{\prime}\right)$ over the photosynthetically active radiation domain (data available at [12]). c: $p_{\boldsymbol{\Omega}, v}^{\mathcal{V}}\left(\boldsymbol{\omega} \mid \boldsymbol{\omega}^{\prime}\right)$ for two wavelengths, where $\theta_{\text {sca }}$ is the angle between $\omega$ and $\omega^{\prime}$.

geometries (using the scientific computation libraries available within the EDStar development environment, see www.starwest.ups-tlse.fr/edstar and [3]). But we keep these technical aspects aside and we concentrate in acceleration potentials related to sensitivity evaluation, in Section 3, and importance sampling in conjunction with the zero-variance methodology, in Section 4. Both require that a complete integral formulation be constructed, strictly reflecting the retained Monte Carlo algorithm. This is the objective of Section 2.

\section{Description of the initial algorithm and construction of the corresponding integral formulation}

We will now design a reverse Monte Carlo algorithm consisting in the sampling of optical paths starting from $\mathbf{x}_{0}$ until they are "absorbed" at the emitting surface $\mathcal{F}$ (see Fig. 1c), in order to evaluate the number of photons $A\left(\mathbf{x}_{0}\right)$ locally absorbed at $\mathbf{x}_{0}$. Each of the corresponding sampling procedures will be detailed hereafter and will be translated into an integral motif. The combination of all these integral motifs will gradually lead us to an integral formulation that is the rigorous formal translation of the proposed algorithm.

(1) First, a frequency is sampled over $\left[v_{\min }, v_{\max }\right]$ according to a uniform probability density function $p_{v}(v)=1 /\left(v_{\max }-v_{\min }\right)$ :

$A\left(\mathbf{x}_{0}\right)=\int_{v_{\min }}^{v_{\max }} p_{v}(v) d v \ldots$

This frequency determines the volume and surfaces radiative properties as well as the surface flux density $\varphi_{v}$ emitted by the fibers $\mathcal{F}$ for the current optical path.

(2) Then, starting from the location $\mathbf{x}_{0}$, a first direction $\omega_{0}$ is sampled over the total solid angle (noted $4 \pi$ hereafter) according to an isotropic probability density function $p_{\mathbf{\Omega}_{0}}^{\mathcal{V}}\left(\omega_{0}\right)=1 / 4 \pi$, and a first scattering length $l_{0}$ is sampled over $[0,+\infty]$ according to the Beer extinction law

$$
\begin{aligned}
p_{L_{0}, v}\left(l_{0} ; k_{s, v}\right)=k_{s, v} \exp \left(-k_{s, v} l_{0}\right): \\
A\left(\mathbf{x}_{0}\right)=\int_{v_{\min }}^{v_{\max }} p_{v}(v) d v \times \int_{4 \pi} p_{\mathbf{\Omega}_{0}}^{v}\left(\omega_{0}\right) d \omega_{0} \\
\quad \times \int_{0}^{\infty} p_{L_{0}, v}\left(l_{0} ; k_{s, v}\right) d l_{0} \ldots
\end{aligned}
$$

(3) Now that $\left\{\mathbf{x}_{0}, \omega_{0}, l_{0}\right\}$ has been sampled, the first interaction location $\mathbf{x}_{1} \equiv \mathbf{x}_{1}\left(\mathbf{x}_{0}, \omega_{0}, l_{0}\right)$ is determined. As previously mentioned pure geometrical considerations are easily translated into scientific computation libraries that we can avoid here to describe. For a given couple $\left\{\mathbf{x}_{0}, \omega_{0}\right\}$, such libraries provide us with the location $\mathbf{y} \equiv \mathbf{y}\left(\mathbf{x}_{0}, \omega_{0}\right)$ of the first time the half line starting at $\mathbf{x}_{0}$ in the direction $\omega_{0}$ intersects the total bounding surface $\mathcal{R} \cup \mathcal{F}$. If the distance to the bounding surface $\left\|\mathbf{y}-\mathbf{x}_{0}\right\|$ is smaller than the scattering length, the optical path interacts with a surface, otherwise scattering occurs inside the volume of culture:

$\mathbf{x}_{1}= \begin{cases}\mathbf{y} & \text { if }\left\|\mathbf{y}-\mathbf{x}_{0}\right\|<l_{0} \\ \mathbf{x}_{0}+l_{0} \omega_{0} & \text { otherwise }\end{cases}$

Then, a branching test is performed depending on the nature of the interaction:

- In case of an interaction with the stainless steel surface of the reactor $\mathcal{R}$, a reflection direction $\omega_{1}$ is sampled according to the diffuse angular distribution $p_{\boldsymbol{\Omega}_{1}}^{\mathcal{R}}\left(\boldsymbol{\omega}_{1}\right)=$ $\left(\omega_{j} . \mathbf{n}_{j}\right) / \pi\left(\mathbf{n}_{j}\right.$ being the normal at the location $\left.\mathbf{x}_{j}\right)$, the weight $\hat{w}$ is multiplied by the reactor reflectivity $\rho^{\mathcal{R}}$ (see the expression of $\hat{w}$ in Eq. (7)), and a new scattering length $l_{1}$ is sampled according to the same extinction law as for $l_{0}\left(p_{L_{1}, v} \equiv p_{L_{0}, v}\right)$ :

$$
\begin{aligned}
& \ldots \times H\left(\mathbf{x}_{1} \in \mathcal{R}\right) \\
& \quad \int_{2 \pi} p_{\mathbf{\Omega}_{1}}^{\mathcal{R}}\left(\omega_{1}\right) d \omega_{1} \int_{0}^{\infty} p_{L_{1}, v}\left(l_{1} ; k_{s, v}\right) d l_{1} \ldots
\end{aligned}
$$

where $H\left(\mathbf{x}_{1} \in \mathcal{R}\right)$ takes the value 1 when the condition $\mathbf{x}_{1} \in \mathcal{R}$ is satisfied, and 0 otherwise. 
- In case of an interaction with the fibers $\mathcal{F}$, a Russian roulette is performed. A random number $r_{1}$ is sampled over the unit interval according to a uniform density probability function $p_{R_{1}}\left(r_{1}\right)=1$. If $r_{1}$ is lower than the fibers reflectivity $\rho^{\mathcal{F}}$, then the optical path is reflected, thus the reflection direction $\omega_{1}$ and $l_{1}$ are sampled as described above $\left(p_{\boldsymbol{\Omega}_{1}}^{\mathcal{F}} \equiv p_{\boldsymbol{\Omega}_{1}}^{\mathcal{R}}\right)$. Otherwise, the optical path sampling procedure is terminated and the weight $\hat{w}_{1}$ is calculated according to Eq. (7). The corresponding integral translation is:

$$
\begin{aligned}
\cdots & \times H\left(\mathbf{x}_{1} \in \mathcal{F}\right) \int_{0}^{1} p_{R_{1}}\left(r_{1}\right) d r_{1} \\
& \left\{\begin{array}{l}
H\left(r_{1} \leq \rho^{\mathcal{F}}\right) \int_{2 \pi} p_{\mathbf{\Omega}_{1}}^{\mathcal{F}}\left(\omega_{1}\right) d \omega_{1} \int_{0}^{\infty} p_{L_{1}, v ; k_{s, v}}\left(l_{1}\right) d l_{1} \ldots \\
+H\left(r_{1}>\rho^{\mathcal{F}}\right) \hat{w}_{1}
\end{array}\right\}
\end{aligned}
$$

- Finally, if $\mathbf{x}_{1}$ is within the volume of culture $\mathcal{V}$, a scattering direction $\omega_{1}$ is sampled according to the single scattering phase function $p_{\boldsymbol{\Omega}_{1}, v}^{\nu}\left(\omega_{1} \mid \omega_{0}\right)$ of Chlamydomonas (see Fig. 2), and $l_{1}$ is sampled as described above:

$$
\begin{aligned}
& \cdots \times H\left(\mathbf{x}_{1} \in \mathcal{V}\right) \\
& \quad \int_{4 \pi} p_{\Omega_{1}, v}^{\mathcal{V}}\left(\omega_{1} \mid \omega_{0}\right) d \omega_{1} \int_{0}^{\infty} p_{L_{1}, v}\left(l_{1} ; k_{s, v}\right) d l_{1} \ldots
\end{aligned}
$$

(4) At this stage, if the path sampling procedure is not terminated, the algorithm loops to (3) considering the next interaction position (the index 1 being incremented to 2 ), and so on until absorption occurs at the fibers.

Altogether, each sampled optical path leads to the evaluation of a weight according to the following weight function $\hat{w}_{j}$, and $A\left(\mathbf{x}_{0}\right)$ is estimated as the average of all weights.

$\hat{w}_{j}=\left(v_{\max }-v_{\min }\right) 4 \pi \sigma_{a, v} \frac{\frac{\varphi_{v}}{h v}}{\pi\left(1-\rho^{\mathcal{F}}\right)}\left[\rho^{\mathcal{R}}\right]^{n_{j}^{\mathcal{R}}} e^{-k_{a, v} d_{j}}$

where $\varphi_{v} / \pi\left(1-\rho^{\mathcal{F}}\right)$ is the equivalent blackbody intensity emitted at the fibers surface, and $\left[\rho^{\mathcal{R}}\right]^{n_{j}^{\mathcal{R}}} e^{-k_{a, v} d_{j}}$ is the absorption transmitivity along the optical path, with $n_{j}^{\mathcal{R}}$ the number of interactions with $\mathcal{R}$ before the $j$ th interaction (the interaction that led to an absorption at $\mathcal{F}$ ) $n_{j}^{\mathcal{R}}=\sum_{q=0}^{j-1}\left[1 \times H\left(\mathbf{x}_{q} \in \mathcal{R}\right)+0 \times H\left(\mathbf{x}_{q} \in \mathcal{F} \cup \mathcal{V}\right)\right]$, and $d_{j}=$ $\sum_{q=0}^{j-1}\left\|\mathbf{x}_{q+1}-\mathbf{x}_{q}\right\|$ representing the total length of the sampled optical path.
The corresponding integral formulation is obtained by gathering all the successive integral motifs of Eqs. (2)-(6): where $\mathcal{A}_{j}$ is recursively defined as

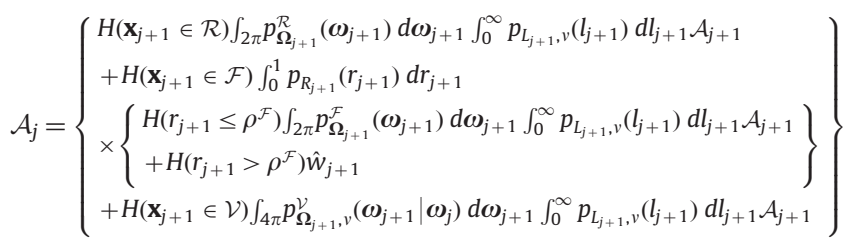

In (4), we will see that modifying this integral formulation is a powerful mean to enhance the convergence of Monte Carlo algorithms. But first, let us illustrate the quite straightforward benefit of integral formulation efforts in the context of sensitivity analysis.

\section{Sensitivity analysis}

When a Monte Carlo algorithm is used for the estimation of a physical quantity $A$, a simple and fast additional procedure can be implemented that simultaneously estimates the partial derivatives of $A$ with respect to any parameter. Such sensitivities of $A$ to a parameter $\pi$ are noted $\partial_{\pi} A$ hereafter. The general methodology consists in deriving the integral formulation of $A$ with respect to $\pi$ and reorganizing it in order to get an integral formulation of $\partial_{\pi} A$ that is identical to that of $A$, replacing only $\hat{w}_{j}$ with a new weight $\hat{w}_{\pi, j}$. Both integral formulations being identical, the sampling events needed for the evaluation of $A$ and $\partial_{\pi} A$ are the same. The required integral formulation tasks range from trivial to very tedious depending on the studied parameter $\pi$ (see [3] for a general overview and [6] for the very specific case of domain deformation sensitivities). Three classically encountered cases are illustrated in this section:

Case 1: Sensitivity to a parameter that only appears in the expression of the weight $\hat{w}$. In the context of photobioreactors study, this can be illustrated through the sensitivity to the absorption properties of the microorganisms. For this purpose, let us introduce the dry mass fraction of pigment $p$ inside the microorganism $(p=0.038$ for Chlamydomonas). As a first approximation, it can be considered that $\sigma_{a, v}=p E_{a, v}$ where $E_{a, v}$ is an effective absorption cross-section of the pigments, and the effect of pigment concentration on the scattering properties can be neglected. Thus, deriving the integral formulation of $A\left(\mathbf{x}_{0}\right)$ with respect to $p$, an integral formulation for $\partial_{p} A\left(\mathbf{x}_{0}\right)$ is obtained that is identical to that of $A\left(\mathbf{x}_{0}\right)$ in Eqs. (8) and

$$
\begin{aligned}
& A\left(\mathbf{x}_{0}\right)=\int_{v_{\min }}^{v_{\max }} p_{v}(v) d v \int_{4 \pi} p_{\mathbf{\Omega}_{0}}^{v}\left(\omega_{0}\right) d \omega_{0} \int_{0}^{\infty} p_{L_{0}, v}\left(l_{0}\right) d l_{0} \\
& \quad \times\left\{\begin{array}{l}
H\left(\mathbf{x}_{1} \in \mathcal{R}\right) \int_{2 \pi} p_{\mathbf{\Omega}_{1}}^{\mathcal{R}}\left(\omega_{1}\right) d \omega_{1} \int_{0}^{\infty} p_{L_{1}, v}\left(l_{1}\right) d l_{1} \mathcal{A}_{1} \\
+H\left(\mathbf{x}_{1} \in \mathcal{F}\right) \int_{0}^{1} p_{R_{1}}\left(r_{1}\right) d r_{1}\left\{\begin{array}{l}
H\left(r_{1} \leq \rho^{\mathcal{F}}\right) \int_{2 \pi} p_{\mathbf{\Omega}_{1}}^{\mathcal{F}}\left(\omega_{1}\right) d \omega_{1} \int_{0}^{\infty} p_{L_{1}, v}\left(l_{1}\right) d l_{1} \mathcal{A}_{1} \\
+H\left(r_{1}>\rho^{\mathcal{F}}\right) \hat{w}_{1} \\
+H\left(\mathbf{x}_{1} \in \mathcal{V}\right) \int_{4 \pi} p_{\mathbf{\Omega}_{1}, v}^{\mathcal{V}}\left(\omega_{1} \mid \omega_{0}\right) d \omega_{1} \int_{0}^{\infty} p_{L_{1}, v}\left(l_{1}\right) d l_{1} \mathcal{A}_{1}
\end{array}\right\}
\end{array}\right\}
\end{aligned}
$$


(9), replacing only $\hat{w}_{j}$ with $\hat{w}_{p, j}$ :

$\hat{w}_{p, j}=\partial_{p} \hat{w}_{j}=\hat{w}_{j} \frac{1-k_{a, v} d_{j}}{p}$

Case 2: Sensitivity to a parameter entering the expression of the sampling probability density functions. In the present context, this can be illustrated with the sensitivity to the biomass density $\eta$, which appears both in $\hat{w}$ and $p_{L_{j}, v}\left(l_{j}\right)$. When deriving Eqs. (8) and (9) with respect to $\eta$, the term corresponding to the derivative of $\hat{w}$ is treated as in case 1 . When deriving $p_{L_{j}, v}\left(l_{j}\right)$, the general form of Eqs. (8) and (9) is temporarily lost. But the obtained expression can be reorganized by multiplying and dividing it by $p_{L_{j}, v}\left(l_{j}\right)$, and reporting the terms $\partial_{\eta} p_{L_{j}, v}\left(l_{j}\right) / p_{L_{j}, v}\left(l_{j}\right)=$ $\left(1-k_{s, v} l_{j}\right) / \eta$ in the final weight function expression. An illustration of this simple reformulation trick is provided hereafter:

$$
\begin{aligned}
\partial_{\eta}[ & \left.\int_{0}^{\infty} p_{L_{0}}\left(l_{0}\right) d l_{0} \int_{0}^{\infty} p_{L_{1}}\left(l_{1}\right) d l_{1} \hat{w}\right] \\
= & \int_{0}^{\infty} p_{L_{0}}\left(l_{0}\right) d l_{0} \int_{0}^{\infty} p_{L_{1}}\left(l_{1}\right) d l_{1} \hat{w}\left[\frac{\partial_{\eta} p_{L_{0}}\left(l_{0}\right)}{p_{L_{0}}\left(l_{0}\right)}+\frac{\partial_{\eta} p_{L_{1}}\left(l_{1}\right)}{p_{L_{1}}\left(l_{1}\right)}\right] \\
& +\int_{0}^{\infty} p_{L_{0}}\left(l_{0}\right) d l_{0} \int_{0}^{\infty} p_{L_{1}}\left(l_{1}\right) d l_{1} \partial_{\eta} \hat{w} \\
= & \int_{0}^{\infty} p_{L_{0}}\left(l_{0}\right) d l_{0} \int_{0}^{\infty} p_{L_{1}}\left(l_{1}\right) d l_{1} \hat{w}\left[\frac{1-k_{s, v} l_{0}}{\eta}\right. \\
& \left.+\frac{1-k_{s, v} l_{1}}{\eta}-\sigma_{a, v} d_{1}\right]
\end{aligned}
$$

In the general case of $j$ interaction locations, this leads to:

$\hat{w}_{\eta, j}=\hat{w}_{j}\left[\sum_{q=0}^{j-1} \frac{1-k_{s, v} l_{q}}{\eta}-\sigma_{a, v} d_{j}\right]$

Case 3: Sensitivity to a parameter appearing in an algorithmic test. Most of the time it corresponds to situations where very little can be done to the date (see [3]), but sometimes an integral reformulation can reduce this complex case to the trivial situation of case 1 . In the present study, this can be illustrated considering the sensitivity to the fibers reflectivity $\rho^{\mathcal{F}}$ which appears both in $\hat{w}$ and in the algorithmic test associated with the Russian roulette (reflection vs. absorption at the fibers). In Eqs. (8) and (9), the integrals involving $r_{j}$ and $\rho^{\mathcal{F}}$ (that correspond to the Russian roulette) have trivial analytical expressions: $\quad \int_{0}^{1} p_{R_{j}}\left(r_{j}\right) d r_{j} H\left(r_{j} \leq \rho^{\mathcal{F}}\right)=\rho^{\mathcal{F}}$ and $\int_{0}^{1} p_{R_{j}}\left(r_{j}\right)$ $d r_{j} H\left(r_{j}>\rho^{\mathcal{F}}\right)=1-\rho^{\mathcal{F}}$. Substituting those expressions into Eqs. (8) and (9), an alternative (but equivalent) integral formulation is obtained (in which the product of all $\rho^{\mathcal{F}}$ is reported in the new weight function expression $\hat{w}^{\text {alt }}$ ):

$\ldots \times H\left(\mathbf{x}_{j} \in \mathcal{F}\right)\left\{\begin{array}{l}\int_{2 \pi} p_{\boldsymbol{\Omega}_{j}}^{\mathcal{F}}\left(\omega_{j}\right) d \omega_{j} \int_{0}^{\infty} p_{L_{j}, v}\left(l_{j}\right) d l_{j} \ldots \\ +\hat{w}_{j}^{\text {alt }}\end{array}\right\}$

where $\hat{w}_{j}^{\text {alt }}=\left[\rho^{\mathcal{F}}\right]^{n_{j}^{\mathcal{F}}}\left(1-\rho^{\mathcal{F}}\right) \hat{w}_{j}$, with $n_{j}^{\mathcal{F}}$ the number of interactions with $\mathcal{F}$ before the interaction at $\mathbf{x}_{j}$ : $n_{j}^{\mathcal{F}}=\sum_{q=0}^{j-1}\left[1 \times H\left(\mathbf{x}_{q} \in \mathcal{F}\right)+0 \times H\left(\mathbf{x}_{q} \in \mathcal{R} \cup \mathcal{V}\right)\right]$ (note the similarity with the treatment of the reflectivity at the surface $\mathcal{R}$ in Eq. (7)). Deriving the above expression with respect to $\rho^{\mathcal{F}}$, we are back to case 1 with $\hat{w}_{\rho^{\mathcal{F}}, j}^{\text {alt }}=$ $\partial_{\rho^{\mathcal{F}}}\left[\hat{w}_{j}^{\text {alt }}\right]=\hat{w}_{j}^{\text {alt }} \frac{n_{j}^{\mathcal{F}}}{\rho^{\mathcal{F}}}$. And we can finally revert the integral transformation to get back to the initial expression that involves a Russian roulette, with:

$\hat{w}_{\rho^{\mathcal{F}}, j}=\hat{w}_{j} \frac{n_{j}^{\mathcal{F}}}{\rho^{\mathcal{F}}}$

Fig. 3 displays the simulation results corresponding to $A\left(\mathbf{x}_{0}\right), \partial_{p} A\left(\mathbf{x}_{0}\right), \partial_{\eta} A\left(\mathbf{x}_{0}\right)$, and $\partial_{\rho^{\mathcal{F}}} A\left(\mathbf{x}_{0}\right)$ as function of the location $\mathbf{x}_{0}$ inside the reactor, for $10^{5}$ realizations (relative standard deviation $\simeq 1 \%$ for all quantities at all locations). The corresponding code is available on demand, and a very similar version is already available at [12]. We distinguish three different zones of the reactor volume [7]: the regions of null production rates (i.e. that the rate of photosynthesis exactly matches the rate of respiration) where $A=A_{c}$ (with the compensation point $A_{c} \simeq 0.5$ mmol s${ }^{-1} \mathrm{~kg}^{-1}$ ), the regions of positive production rates (photosynthesis) where $A>A_{c}$, and the regions of negative production rates (respiration) where $A<A_{c}$. The studied prototype is designed to approach the
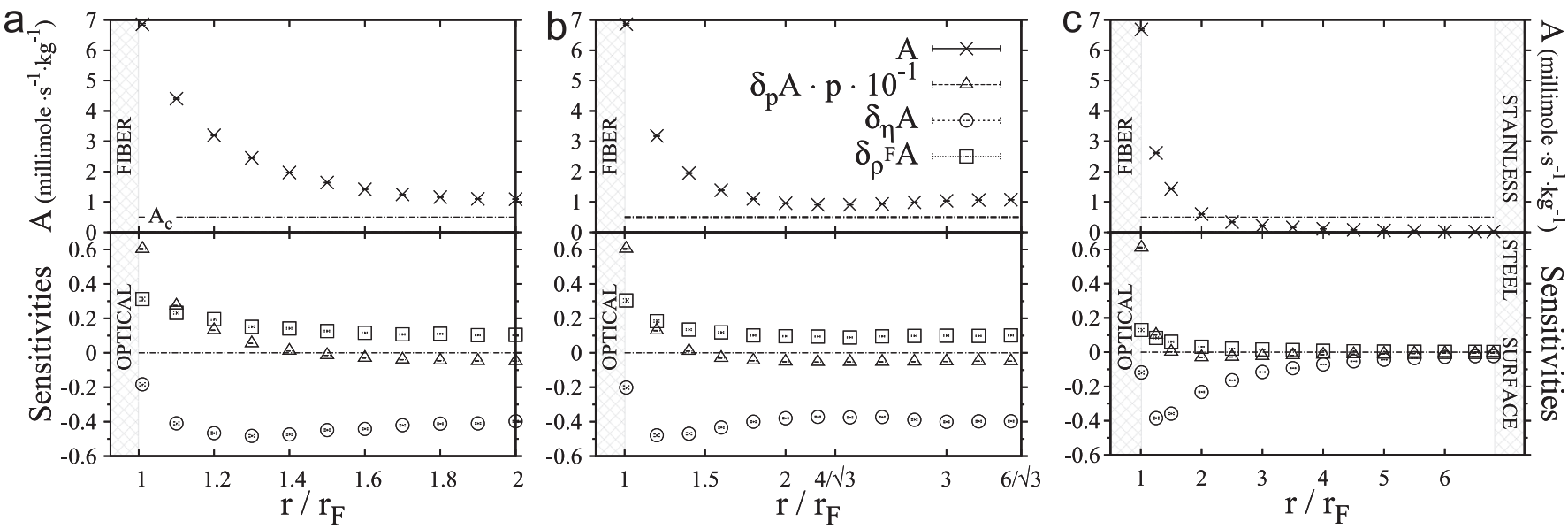

Fig. 3. Simulation results for $A\left(\mathbf{x}_{0}\right)$ and its sensitivities $\partial_{p} A, \partial_{\eta} A$ and $\partial_{\rho^{F}} A$ as defined in Eqs. (8) and (9), with $\varphi_{v}=10^{-7}\left(2 \pi v^{2} / c^{2}\right)$ exp[ $\left.-\left(h v / K_{B} 6000-1\right)\right]$ ( $\propto$ Planck's law at $6000 \mathrm{~K}$ ), $\rho^{\mathcal{F}}=0.1, \rho^{\mathcal{R}}=0.5, \eta=4 \mathrm{~kg} \mathrm{~m}^{-3}$. $\mathbf{x}_{0}$ referred with $(r, \theta)$ as defined in Fig. 1 . In a and b the fiber is at the center of the reactor and in $\mathrm{c}$ the fiber is at the edge of the reactor. (a) $\theta=0$; (b) $\theta=\pi / 6$; (c) $\theta=0$. 
maximum efficiency of photosynthesis (at the expense of its volume productivity, see [8-10]) which is ideally achieved when the whole reactor volume corresponds to low productions rates (i.e $A\left(\mathbf{x}_{0}\right)$ uniform at a value close to $\left.A_{c}\right)$. Nevertheless, the results of $A\left(\mathbf{x}_{0}\right)$ indicate high production rates near by the fibers ( $c f$. $\mathrm{a}$ and $\mathrm{b}$ ) and respiration regions between the edge fibers and the outside surface of the reactor ( $c f$. c). The microorganisms density $\eta$ is the main operational parameter, but the field of $\partial_{\eta} A\left(\mathbf{x}_{0}\right)$ indicates a weak influence of $\eta$ within the respiration zones, suggesting a design optimization consisting in a reduction of the distance between the edge fibers and the reactor. The field of $\partial_{\rho^{\mathcal{F}}} A\left(\mathbf{x}_{0}\right)$ suggests another design optimization consisting in the choice of reflecting fiber materials limiting the losses and thus allowing an operation at higher densities $\eta$ (i.e. an intensification). Finally, the field $\partial_{p} A\left(\mathbf{x}_{0}\right)$ indicates, as pointed out by the genetic engineering community [11], that a diminution of the pigment content of microorganisms leads to an homogenization of $A\left(\mathbf{x}_{0}\right)$, but the overall energy absorbed by the culture decreases and losses at the fibers and the reactor surface increase.

\section{Convergence enhancement}

Various techniques are available in order to reduce the standard deviation of the set of sampled weights, for a fixed number of realizations. Most such convergence enhancement tasks actually consist in the injection of our physical understanding of the system into the Monte Carlo algorithm, in order to privilege the events (or informations) that are important with respect to the estimated quantity (see [1-3]). Two of those techniques will be illustrated in the following (thanks to some of the recent integral formulation and zero-variance perspectives): importance sampling, and integral reformulation.

Importance sampling consists in working on the sampling procedures. Let us consider the first two sampling procedures, corresponding to $p_{v}$ and $p_{\Omega_{0}}^{v}$ in the integral formulation of Eqs. (8) and (9). A modification of these sampling procedures is very easy to implement: $p_{v}$ and $p_{\Omega_{0}}^{\mathcal{V}}$ are replaced with better optimized probability density functions $p_{v, o p t}$ and $p_{\boldsymbol{\Omega}_{0}, o p t}^{\mathcal{V}}$ and the weight function is replaced by a new expression $\hat{w}_{o p t}=\hat{w}\left(p_{v} / p_{v, o p t}\right) p_{\Omega_{0}}^{\nu} /$ $p_{\Omega_{0}, o p t}^{\mathcal{V}}$ that insures the evaluation of the same exact solution. However, the choice of new probability density functions that would lead to a convergence enhancement is commonly very subtle, and we argue here that the concept of zero-variance is an efficient way to orient this choice. Zero-variance algorithms are such that for each event the weight value is strictly identical to $A\left(\mathbf{x}_{0}\right)$ itself. This means that the sampling procedures have been chosen such that only one single event is required to get a perfect estimator. Let us illustrate this concept, as developed in $[4,5]$, on the sampling procedure of $v$. The integral formulation of Eqs. (8), (9) and (7) can be rewritten in the compact form:

$A\left(\mathbf{x}_{0}\right)=\int_{v_{\min }}^{v_{\max }} p_{v, o p t}(v) d v A\left(\mathbf{x}_{0}, v\right) \frac{1}{p_{v, o p t}(v)}$ where $A\left(\mathbf{x}_{0}, v\right) 1 / p_{v, o p t}(v)$ represents the rest of the integral formulation, with $A\left(\mathbf{x}_{0}, v\right)$ having a simple physical meaning that is obvious from Eq. (1): $A\left(\mathbf{x}_{0}, v\right)$ is the specific number of photon absorbed per unit frequency i.e. $A\left(\mathbf{x}_{0}, v\right)=\left(\sigma_{a, v} / h v\right) \int_{4 \pi} L_{v}\left(\mathbf{x}_{0},-\omega_{0}\right) d \omega_{0}$, where $\int_{4 \pi} L_{v}\left(\mathbf{x}_{0}\right.$, $\left.-\omega_{0}\right) d \omega_{0}$ is the local monochromatic irradiance $G_{v}\left(\mathbf{x}_{0}\right)$. Zero-variance algorithm on the integration of $v$ is thus achieved for:

$p_{v, o p t}(v)=\frac{A\left(\mathbf{x}_{0}, v\right)}{\int_{v_{\min }}^{v_{\max }} A\left(\mathbf{x}_{0}, v\right) d v}$

Indeed, in this case, whatever the sampled frequency value, the weight $A\left(\mathbf{x}_{0}, v\right) 1 / p_{v, \text { opt }}(v)=\int_{v_{\min }}^{v_{\max }} A\left(\mathbf{x}_{0}, v\right) d v=$ $A\left(\mathbf{x}_{0}\right)$. We therefore see that the design of a zero-variance algorithm for the estimation of $A\left(\mathbf{x}_{0}\right)$ requires an a priori perfect knowledge of $A\left(\mathbf{x}_{0}, v\right)$ for all $v$, and of its integral over $v$, that is to say of $A\left(\mathbf{x}_{0}\right)$ itself. This means that such an ideal algorithm can only be practically implemented when it is useless (when $A\left(\mathbf{x}_{0}\right)$ is known). Nevertheless, zero-variance helps us to understand the physical information required to ideally optimize a given sampling procedure, here the irrandiance $G_{v}\left(\mathbf{x}_{0}\right)$, and if this information is accessible under any approximate form, an enhanced version of the algorithm can be designed in a straightforward manner. The idea here is not to get a "good approximation" of $G_{v}\left(\mathbf{x}_{0}\right)$ but only a model that grasps the variations of $G$ with respect to $v$ in order to drive the sampling procedure. Let us make a first simple attempt in which the probability density function is obtained by replacing $A\left(\mathbf{x}_{0}, v\right)$ in Eq. (16) using the spectral dependence of $G_{v}\left(\mathbf{x}_{0}\right)$ provided by the P1 approximation. Fig. 4a indicates that the corresponding convergence enhancement is not significant. The reason can either be that:

1. The frequency dependence is well grasped by the P1 approximation but frequency sampling is not a significant source of variance (in which case further investigations can for instance concentrate on the next sampling procedure, as illustrated later in this text).

2. The P1 approximation was too rough to provide enough information concerning the frequency dependence of $A\left(\mathbf{x}_{0}, v\right)$.

If we assume the second reason to be meaningful, then either we look for a better irradiance model, or we try to make use of integral reformulation.

Integral reformulation consists in transforming the integral formulation in such a way that the quantity highlighted by the zero-variance approach (which is to be closely studied in order to design a better optimized probability density function) has a clear physical meaning and can be easily modeled. One of the simplest way to perform this task (when applicable) is to report the studied integration (here the integration over $v$ ) at a rank of the integral formulation such that the remaining physics (i.e. the following integrations) is easier to handle. As a very ideal example, we report hereafter the spectral integration at the end of the integral formulation (i.e. $p_{v}(v)$ becomes the last sampling procedure). Importance 

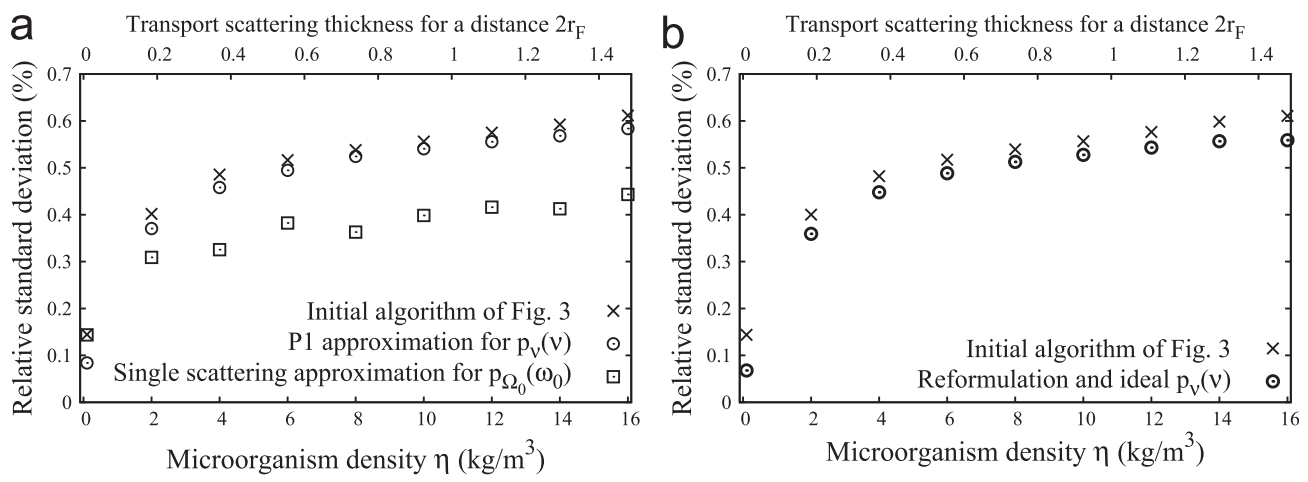

Fig. 4. Convergence of the $A\left(\mathbf{x}_{0}\right)$ estimator for $10^{5}$ realizations at the center of the reactor $\left(r=1.2 r_{\mathcal{F}}, \theta=\pi / 12\right)$. (a) Importance sampling for $v$ and $\mathbf{\Omega}_{0}$. (b) Integral reformulation and ideal importance sampling for $v$ using gray scattering properties (properties of Fig. 2 at $4.5 \times 10^{14} \mathrm{~Hz}$ ).

sampling can then be implemented without the need of any multiple-scattering model. This is immediately possible as soon as Chlamydomonas is approximated as having gray scattering properties (i.e. $p_{\boldsymbol{\Omega}_{j>0}, v}^{\mathcal{V}} \simeq p_{\boldsymbol{\Omega}_{j}, e q}^{\mathcal{V}}$ and $\left.p_{L_{j}, v} \simeq p_{L_{j}, e q}\left(l_{j}\right)\right)$, spectral absorption properties remaining unchanged. This way, all dependences to $v$ are in the weight and the integral formulation of Eqs. (8) and (9) is easily reformulated as:

$A\left(\mathbf{x}_{0}\right)=\int_{4 \pi} p_{\mathbf{\Omega}_{0}}^{v}\left(\omega_{0}\right) d \omega_{0} \int_{0}^{\infty} p_{L_{0}, e q}\left(l_{0}\right) d l_{0} \ldots \int_{v_{\min }}^{v_{\max }} p_{v}(v) d v \hat{w}_{j}$

with $\hat{w}_{j} \propto\left(\varphi_{v} / h v\right) \sigma_{a, v} e^{-k_{a, v} d_{j}}$ (see Eq. (7)). The ideally optimized probability density function is then $p_{v}^{I}(v)=$ $\left(\varphi_{v} / h v\right) \sigma_{a, v} e^{-k_{a, v} d_{j}} / \int_{v_{\min }}^{v_{\max }}\left(\varphi_{v} / h v\right) \sigma_{a, v} e^{-k_{a, v} d_{j}} d v$. Although less straightforward, the same approach can be used accounting for the spectral scattering properties: the multiple scattering/reflection optical path is sampled using any gray reference scattering coefficient and phase function, the weight expression being corrected, once frequency is finally sampled, by the product of the ratios of the exact scattering probability density function to the reference one. Fig. $4 \mathrm{~b}$ shows that even in the case of an ideal optimization of the frequency sampling procedure, the convergence is only little enhanced. We now know precisely that we are in the first of the two above identified cases: frequency is not a significant source of variance.

We therefore further investigate the convergence enhancement opportunities by considering the next sampling procedure: the sampling of the first direction $p_{\mathbf{\Omega}_{0}}^{\mathcal{V}}$. The same zero-variance technique as in Eqs. (15) and (16) leads here to:

$$
\begin{aligned}
A\left(\mathbf{x}_{0}\right)= & \int_{v_{\min }}^{v_{\max }} p_{v, o p t}(v) d v \int_{4 \pi} p_{\mathbf{\Omega}_{0}, o p t}^{\nu}\left(\omega_{0}\right) d \omega_{0} \\
& \times A\left(\mathbf{x}_{0}, v, \omega_{0}\right) \frac{1}{p_{v, o p t}(v) p_{\boldsymbol{\Omega}_{0}, o p t}^{\nu}\left(\omega_{0}\right)}
\end{aligned}
$$

with

$p_{\Omega_{0}, o p t}^{\mathcal{\nu}}\left(\omega_{0}\right)=\frac{A\left(\mathbf{x}_{0}, v, \omega_{0}\right)}{\int_{4 \pi} A\left(\mathbf{x}_{0}, v, \omega_{0}\right) d \omega_{0}}=\frac{A\left(\mathbf{x}_{0}, v, \omega_{0}\right)}{A\left(\mathbf{x}_{0}, v\right)}$

where $A\left(\mathbf{x}_{0}, v, \omega_{0}\right)$ is the specific number of photons absorbed at $\mathbf{x}_{0}$, per unit frequency and (now) per unit solid angle in the direction $\omega_{0}$ i.e. $A\left(\mathbf{x}_{0}, v, \omega_{0}\right)=\left(\sigma_{a, v} / h v\right)$
$L_{v}\left(\mathbf{x}_{0},-\omega_{0}\right)$ (see Eq. (1)). It is now an angular dependence, for a given frequency, which we try to grasp using an approximate model. In our first frequency optimization attempt, we made use of the P1 approximation. This was weakly justified considering our intermediate optical thicknesses but we needed to deal with an angular integrated quantity and P1 was the best approach we could think of. Here we can do much better using the single scattering approximation. Fig. 4a shows that the corresponding convergence enhancement leads to a reduction of the weight standard deviation of a factor $\frac{2}{3}$, which divides by $\left(\frac{3}{2}\right)^{2} \simeq 2$ the number $N$ of required samples (to achieve the same convergence) by comparison with our first algorithm (the standard deviation of a Monte Carlo algorithm being proportional to $1 / \sqrt{N}$ ).

Note that the P1 and single scattering models [13] have been built considering an infinite periodic fiber arrangement (see the elementary configuration of Fig. 1c in which the surfaces noted ... are perfect specular mirrors). The geometry is even further simplified using an equivalent $1 \mathrm{~d}$ cylindrical geometry for the P1 approximation and an equivalent slab for the single scattering approximation. Altogether, the zero-variance approach incites us to elaborate gradually simplified models at various description scales, and this is in this sense that such a practice leads to useful physical pictures, although the initial objective was only acceleration.

\section{Conclusion}

The integral formulation tasks presented here illustrate our practice of radiative transfer Monte Carlo methods. Along this line, we laid the foundations of a subsequent energetic analysis of photobioreactors (which will necessitate the use of volume integrated growth kinetics models). This approach already allows us to accurately estimate the absorption field and its sensitivities (except for domain deformation sensitivities) within a geometrically complex volume of culture. Direction sampling appears as the source of variance that is worth the closest attention. Using only a rough one-dimensional simplification of the geometry in order to implement importance sampling, convergence was significantly enhanced, indicating that higher speedups can be expected by precomputing and tabulating the angular 
dependences as function of optical thickness and fibers lattice features.

\section{Acknowledgments}

The research presented in this paper was partially conducted with the support of the ITAAC project (Impact du Trafic Aérien sur l'Atmosphére et le Climat), funded by the Fondation Sciences et Technologies pour l'Aéoronautique et l'Espace (STAE), Toulouse, France, within the Réseau Thématique de Recherche Avancée (RTRA). This work was also partially supported by the French ANR agency through the PNRB program BIOSOLTS (http:// www.biosopis.org).

\section{References}

[1] Howell JR. J Heat Transfer 1998;120(3):547-60.

[2] Modest MF. Radiative heat transfer. second ed.Academic Press; 2003.

[3] De la Torre J, et al. Sol Energy, Submitted for publications.

[4] Assaraf R, Caffarel M. Phys Rev Lett 1999;83(23):4682-5.

[5] Hoogenboom JE. Nucl Sci Eng 2008;160(1):1-22.

[6] Roger M, Blanco S, El Hafi M, Fournier R. Phys Rev Lett 2005;95: 180601.

[7] Cornet JF, Dussap CG. Biotechnol Prog 2009;25(2):424-35.

[8] Cornet JF. Chem Eng Sci 2010;65(2):985-98.

[9] Csogor Z, Herrenbauer M, Schmidt K, Posten C. J Appl Phycol 2001; $13: 325-33$

[10] Suh IS, Lee SB. Biotechnol Bioeng 2003;82:180-9.

[11] Pilon L, Berberoglu H, Kandilian R. J Quant Spectrosc Radiat Transfer 2011:112:2639-60

[12] <www.starwest.ups-tlse.fr/ monte-carlo-concentrated-solar-examples $\rangle$.

[13] Case KM, Zweifel PF. Linear transport theory.Reading, Massachusetts: Addison-Wesley; 1967. 